\title{
Evaluation of Consultation Notes Within and Across Institutions: A Preliminary Study
}

Amee Sangani ${ }^{1}$, April Savoy ${ }^{2}$

${ }^{1}$ Indiana University School of Medicine, ${ }^{2}$ Purdue School of Engineering and Technology

Background/Objective: Patients with multiple chronic conditions require specialty consultations both within and across institutions for effective co-management of comorbidities. Poor communication during the referral process increases physician workload, patient burden and risks. Successful co-management relies on bi-directional information flow that supports interpersonal communication and establishment of clear tasks and responsibilities among physicians. However, flow of health information is often limited to specific health network access, phone calls, or faxes. Interpersonal communication is dependent on limited encounter notes. In this preliminary study, our objective was to understand how consultants' notes support physician collaboration within and across health care institutions.

Project Methods: To assess consultants' notes, outpatient charts were randomly selected from the Indiana Network for Patient Care database representing consultations with five different specialties within the IU Health network, including referrals from within and outside of IU Health. The Quality of Consult Assessment tool was adapted to assess content of notes, emphasizing clinical recommendations, distribution of tasks and responsibilities, and communication plans.

Results: Our sample included ten charts for patients who had comorbidities. All notes contained clinical recommendations that included an assessment and plan. $70 \%$ of notes contained explicit responsibilities of the consultants. Conversely, only one contained explicit responsibilities for referrers. Charts denoted reliance on support staff to send messages between referrers, consultants, and patients via phone and fax. Phone calls and faxes were more prominent in referrals across institutions.

Conclusion and Impact: Our preliminary findings indicate that current clinical documentation supports specialty referrals for transitions of care rather than co-management of care.

Difficulties in accessing patient charts across institutions leads to a lack of clinical context and workflow inefficiencies when attempting to co-manage care. These findings demonstrate negative implications in health outcomes for patients with multiple comorbidities that require more care coordination within and across institutions. 